\title{
Diseño y evaluación de un programa Mindfulness para estudiantes de Arte Dramático
}

\author{
Design and evaluation of a program Mindfulness for students of dramatic art
}

\author{
Olga Magaña, Margarita Pino-Juste \\ Universidad de Vigo
}

\begin{abstract}
Resumen
El trabajo se centra en el diseño y evaluación de un programa basado en mindfulness a través de un estudio de caso realizado con el alumnado de segundo curso de la especialidad de Interpretación en la titulación de Arte Dramático. Se evalúa la efectividad del programa sobre los niveles de autoconciencia, empatía y creatividad. La intervención psicoeducativa tiene una duración de ocho semanas de entrenamiento. Se describen algunas actividades básicas del programa compuesto por técnicas de expresión oral y corporal y prácticas de memoria sensorial, así como los resultados obtenidos. Se utiliza un diseño pre-test, post-test para medir la eficacia del mismo.
\end{abstract}

Palabras clave: mindfulness, educación, autoconciencia, creatividad, arte dramático, empatía.

\begin{abstract}
The work is centred on the design and evaluation of a program which is based in Mindfulness with pupils of the second course of the speciality of Interpretation of the studies of Dramatic Art. It is a question of a study of a case where one tries to measure the efficiency of the program on the levels of self-conciusness, empathy and creativity. The psychoeducational intervention has got a duration of eight weeks of training. There are thence described some basic activities of the program, composed by oral and corporal expression techniques, and examples of sensory memory reports, as well as the aims or objectives that we have tried to get reached. It is both used a pre-test, post-test design in order to measure the efficiency of the very same one.
\end{abstract}

Key Words : mindfulness, education, self-conciousness, creativity, dramatic art, empathy.

\section{Marco teórico}

El objetivo de este trabajo ha sido diseñar y evaluar un programa basado en Mindfulness para el fomento de la empatía, la autoconciencia y la creatividad en futuros actores. Entre las competencias más relevantes que el actor debe alcanzar en su formación, figuran comprender, entender y sentir las vidas, situaciones y personalidades ajenas. Para ello necesitará auto regular sus emociones, identificarlas y aprender a expresarlas. Deberá usar de manera eficaz sus capacidades de imaginación, intuición, inteligencia emocional y pensamiento creativo para la solución de problemas, así como su habilidad para pensar y trabajar con flexibilidad. "El actor es a su vez objeto y sujeto de su propio trabajo, por ello la pedagogía actoral deberá tener presente este proceso autogestor" (Serrano, 2004, p.23).

La formulación del modelo de autoconciencia de Scheier y Carver (1985) se centra en la disposición o tendencia de las personas a focalizar su atención sobre sí mismos, ya sea sobre sus aspectos privados, (aspectos internos, sentimientos, estados de ánimo, pensamientos, etc.) o sobre sus aspectos públicos (aspectos externos, aspecto físico, impresión en los demás etc.). Siendo el individuo su propio objeto de atención (Duval \& Wicklund, 1972), el desarrollo de la autoconciencia resulta un camino a seguir para la gestión de las emociones. Por otra parte Davis (1994 p.12) define la empatía como "conjunto de constructos que incluyen los procesos de ponerse en el lugar del otro y respuestas afectivas y no afectivas". En cuanto a la creatividad, algunos estudios consideran la capacidad de observar asociada a un aumento de la flexibilidad cognitiva (Chambers, Gullone, \& Allen, 2009) y que dicha flexibilidad es un motor clave en los procesos creativos (De Dreu, Nijstad, Baas, Wolsink, \& Roskes, 2012).

El mindfulness se ha definido por Kabat- Zinn como "la conciencia que emerge a través de poner atención intencional, en el momento presente, de manera no condenatoria, del flujo de la experiencia momento a momento" (Kabat-Zinn, 2005 p.145). Las investigaciones realizadas en el campo de la educación subrayan que la práctica de la atención plena mejora el rendimiento académico y las habilidades cognitivas (Carboni, 2012); incrementa la regulación emocional y los estados positivos de la mente (Baer, Smith, Hopkins, Krietemeyer, \& Toney, 2006), reduce el stress académico (Sibinga et al., 2013) y mejora el aprendizaje (Brown \& Ryan, 2003). La técnica de Mindfulness está siendo utilizada además como instrumento para el incremento de la creatividad (Baas, Nevicka, \& Ten Velden, 2014).

La inclusión de la Enseñanzas Artísticas Superiores en el Espacio Europeo de Educación Superior (EEES) apuesta por una educación basada en competencias (conocimientos, habilidades y valores) que el alumno debe adquirir durante su formación. En relación a las competencias que el alumno debe desarrollar en el área artística, Gadner (Gardner \& Nogués, 1998) señala tres tipos de competencias: la producción de composiciones artísticas, la percepción asociada a la habilidad para efectuar distinciones o discriminaciones dentro de una forma artística y la reflexión o capacidad para alejarse de sus propias 
percepciones y producciones comprendiendo los objetivos, los métodos y los procesos para alcanzar los efectos conseguidos. Desde este punto de vista podríamos concluir que en su formación, el futuro actor/actriz debe aprender a investigar su singularidad creativa y ser capaz de establecer criterios selectivos que le permitan utilizar las estrategias metodológicas más adecuadas para llevar a cabo su obra. El entrenamiento actoral comienza por desarrollar capacidades relacionadas con tomar consciencia corporal, vocal y emocional. Aunque existen diversas técnicas que posibilitan el desarrollo de dichas habilidades, no existen programas específicos que unifiquen el desarrollo de las mismas de forma sistemática.

Las principales teorías relativas a la formación actoral están ligadas a la interacción mente- cuerpo y se fundamentan en la necesidad de comprometer el cuerpo y la mente en un acto de voluntad consciente. El llamado "sistema Stanislavski" es el referente obligado de la pedagogía teatral del siglo XX. Propone una metodología basada en la “experienciación” y relaciona el término con estados mentales que predisponen hacia una activación de la creatividad. Partiendo de que la tensión física paraliza el cuerpo e interfiere la habilidad mental, sugiere que los actores ejerciten la respiración y propone la práctica del "Hatha Ioga” para posibilitar hábitos de relajación que les permitan desarrollar la concentración y la imaginación (Carnicke, 2000). En relación a la gestión de emociones, Meyerhold (1975) propuso la observación de la relación de condicionamiento que existe entre los estados de ánimo psicológicos y los procesos de tipo fisiológico con el objetivo de que el actor lograra coordinar su interpretación sin ceder al impulso de la emocionalidad. La Autorregulación emocional, según Siegel (2015) es una habilidad de la mente que consiste en modificar en distintos niveles el proceso emocional.

\section{Método}

\section{Participantes}

La muestra del presente estudio se compone de 37 estudiantes de la Escuela Superior de Arte Dramático de Galicia, con una edad comprendida entre los 18 y los 29 años. El grupo experimental estuvo formado por 20 sujetos (6 hombres y 14 mujeres) de segundo curso de Interpretación y el grupo control por 17 estudiantes (9 hombres y 8 mujeres) de tercer curso de Interpretación.

\section{Instrumentos}

Se ha utilizado un paradigma crítico mediante un estudio de caso grupal. Los instrumentos de análisis son de carácter cuantitativo (escalas para medir cada una de las variables). Se utiliza un diseño pretest-postest para medir la eficacia del programa. Para la medición de constructos se han aplicado los siguientes instrumentos:

La variable empatía se ha medido a través de la Escala EDI de empatía e identificación con los personajes (Rovira \& Perosanz, 1998) así como mediante la escala
ECSE de evaluación de Competencias Socioemocionales que se describe en párrafos posteriores.

La escala EDI se compone de 17 ítems que el sujeto contesta tras el visionado de fragmentos de diferentes películas. El formato de respuesta es de cinco puntos (donde 1 significa «nada» y 5 significa «mucho»). A mayor puntuación mayor identificación con los personajes. La consistencia interna de la escala EDI para los segmentos fílmicos negativos es alta, con coeficiente alpha de Cronbach de .90 y posee una satisfactoria fiabilidad o consistencia interna, para ambos estímulos.

Se han utilizado dos segmentos fílmicos elaborados a partir de la selección de determinadas escenas de diferentes películas. El segmento positivo contenía una serie de situaciones seleccionadas de escenas cómicas de cámara oculta y su duración era de 5 minutos y 20 segundos. Aparecían diversas situaciones graciosas en las que distintas personas sufrían una serie de bromas. El segmento negativo se componía de escenas de diversas películas de aventuras y/o terror ("En busca del arca perdida”, "La vida de Pi”, "Resident Evil”, "Kill Bill 2", "La morada del miedo"); su duración era de 5 minutos y 44 segundos. En él aparecían imágenes que se podrían describir como desapacibles. Al llegar a la sala se leyeron las instrucciones. Después de la visión de los fragmentos positivos, cumplimentaron la escala de identificación con los personajes. A continuación se leyeron nuevamente las instrucciones y visionaron el segundo segmento negativo y contestaron de nuevo el cuestionario.

Para la evaluación de la creatividad se aplicó el Test de Inteligencia Creativa CREA (Corbalan, Martínez, \& Donolo, 2003) El test utiliza como procedimiento de medida de la creatividad del sujeto su capacidad para elaborar preguntas. La tarea consiste en escribir brevemente cuantas preguntas sean posibles hacer sobre lo que representa una ilustración que se le entrega dentro del cuadernillo en un tiempo limitado. Corbalán et. al. (2006) reportan que el instrumento presenta confiabilidad adecuada establecida a través del método de las formas paralelas donde se obtuvo una fiabilidad de .875 . En lo relativo a la validez se reporta adecuada validez predictiva y concurrente con el Test de Guilford, y validez discriminante ya que la prueba permite diferenciar a los grupos de baja y alta creatividad.

Nos inclinamos a utilizar esta prueba porque nos permite evaluar no los logros alcanzados sino el equipamiento cognitivo del sujeto en relación con su productividad creativa.

Se aplicó además la Escala de evaluación de Competencias Socioemocionales ECSE (Repetto et al., 2009). Dicha escala, con fiabilidad Cronbach .895, consta de 38 ítems agrupados en siete sub-escalas de tipo auto-informe, que miden cada una de ellas una competencia socioemocional concreta. Las siete competencias socioemocionales son las siguientes: autoconciencia, regulación emocional, inter.-personal, empatía, motivación, trabajo en equipo y resolución de conflictos. El sujeto debe señalar el grado en el que se identifica con la afirmación contenida en él, dentro de 
una escala Likert de 5 puntos: nada identificado, poco identificado, ocasionalmente identificado, bastante identificado, totalmente identificado.

\section{Descripción del programa}

La técnica Mindfulness se presenta como intervención psicoeducativa para la mejora de la autoconciencia, la empatía y la creatividad. El criterio a seguir para la selección de actividades fue elegir, por una parte aquellas relacionadas con la pedagogía teatral (Carnicke, 2009; Chejov, Ferrigno, \& Lizarraga, 1987; Chéjov, 1999; Layton \& Esteve, 1995; Motos Teruel \& Tejedo, 1987; Serrano, 2004; Stanislavski, 2013) y por otra, aquellas que fomentan la atención plena (Asuero, 2009; Kabat-Zinn, 1994) y la formación en competencias socioemocionales (Repetto, 2009). Algunas fueron diseñadas en base a experiencias con actores dentro del ámbito docente y otras pertenecen a los autores mencionados, rescatando la idea original o buscando variaciones de las mismas.

El programa tiene una duración de ocho semanas, distribuidas en sesiones semanales de dos horas y media

\section{Competencias y objetivos}

A continuación detallamos las competencias y sus objetivos relacionados (Tabla 1)

Tabla 1

Objetivos agrupados por competencias

\begin{tabular}{ll}
\hline \multicolumn{1}{c}{ Competencias } & \multicolumn{1}{c}{ Objetivos específicos } \\
\hline Autoconciencia y capacidad de atención & $\begin{array}{l}\text { Diferenciar estados emocionales a través del lenguaje no verbal } \\
\text { Aprender a expresar sentimientos, emociones y pensamientos } \\
\text { Reproducir comportamientos y recrear en escena situaciones } \\
\text { imaginarias } \\
\text { Aprender a regular y gestionar emociones con flexibilidad }\end{array}$ \\
\hline Empatía y adaptación a roles & Desarrollar la capacidad de escucha y reaccionar adecuadamente a \\
& estímulos \\
& Aprender a reconocer y expresar de forma adecuada las emociones \\
& propias y ajenas \\
& Manejar las claves del lenguaje verbal y no verbal \\
& Construir y crear personajes en situaciones imaginarias \\
\hline Habilidades de comunicación consciente & Estimular el impulso creativo \\
& Potenciar recursos expresivos: voz, gesto y palabra \\
& Comunicar con eficacia y fluidez. \\
& Eliminar bloqueos comunicativos \\
& Adquirir presencia escénica. \\
\hline
\end{tabular}

\section{Actividades}

A continuación presentamos una tabla descriptiva (Tabla 2) como ejemplo de actividades por sesión.

Tabla 2

Actividades, competencias y temporalización

\begin{tabular}{ll}
\hline ACTIVIDAD n $^{\mathbf{0}} \mathbf{1 :}$ & Inventario corporal \\
\hline Competencias & Autoconciencia y capacidad de atención \\
Temporalización: & 25 minutos \\
Descripción: & Recostados en el suelo. Recorrido corporal y relajación guiada. \\
\hline ACTIVIDAD n $\mathbf{n}^{\mathbf{2}}$ & Exploración de sonidos \\
\hline Competencias & Autoconciencia y capacidad de atención \\
Temporalización: & Habilidades de comunicación consciente \\
Descripción: & 15 minutos \\
\hline ACTIVIDAD n & Atención focalizada en la respiración diafragmática. Sonorización de textos breves \\
\hline
\end{tabular}

y una última sesión de cuatro horas. Está dividido en dos bloques: las prácticas formales dentro del aula (individuales y colectivas) y las prácticas informales fuera del aula (individuales).

Prácticas formales. Cada una de las sesiones se estructura de manera similar: 1. Lectura de un texto (poema, monólogo o escena) o visionado de fragmentos de películas u obras de teatro en relación con las tres competencias a trabajar. 2. Presentación de contenidos teóricos. 3. Práctica de actividades y 4. Puesta en común.

Prácticas informales. Están compuestas de una serie de prácticas que realiza el alumno diariamente durante sus actividades cotidianas: caminar prestando atención, comer conscientemente percibiendo sensaciones, conversar prestando atención a las reacciones y emociones, escuchar al interlocutor y planificar y gestionar el tiempo, entre otras. Las prácticas pueden tener una duración que oscila entre cinco minutos y quince minutos. 


\begin{tabular}{|c|c|}
\hline Competencias & Autoconciencia y capacidad de atención \\
\hline Temporalización: & 20 minutos \\
\hline Descripción: & Memoria sensorial. Inducción a imágenes internas espontáneas a través de aromas. \\
\hline ACTIVIDAD n ${ }^{\circ}$ : & Paseo a ciegas \\
\hline Competencias & Empatía y adaptación a roles. \\
\hline Temporalización: & 20 minutos \\
\hline Descripción: & $\begin{array}{l}\text { Por parejas. “A” con los ojos vendados es guiado por “ B” en un trayecto. } \\
\text { Cambio de roles. }\end{array}$ \\
\hline ACTIVIDAD n ${ }^{0}$ : & La despedida \\
\hline Competencias & $\begin{array}{l}\text { Habilidades de comunicación consciente. } \\
\text { Empatía y adaptación a roles. }\end{array}$ \\
\hline Temporalización: & 25 minutos. \\
\hline Descripción: & Improvisación por parejas en torno a un conflicto. “A” quiere algo de “B” y “B” niega. \\
\hline ACTIVIDAD n ${ }^{\circ}$ 6: & Sucesos \\
\hline Competencias & $\begin{array}{l}\text { Autoconciencia y capacidad de atención } \\
\text { Habilidades de comunicación consciente }\end{array}$ \\
\hline Temporalización: & 40 minutos \\
\hline Descripción: & $\begin{array}{l}\text { En parejas "A “cuenta un suceso y " } B \text { " escucha. Posteriormente "B" intenta repetir con exactitud } \\
\text { las palabras y gestos de "A" al contar el suceso. }\end{array}$ \\
\hline
\end{tabular}

\section{Resultados}

Tras seguir las normas de corrección de las pruebas y obtener las puntuaciones directas, se realizaron diversos cálculos de estadística descriptiva: media y desviación típica.

La Tabla 3 muestra las medias y desviaciones típicas pre-test y pos-test correspondientes a los grupos experimental y de control para las variables de autoconciencia, empatía y creatividad.

Para comprobar si se habían producido mejoras significativas en el grupo experimental para las tres variables de estudio una vez finalizada la intervención, se realizó un análisis de las diferencias entre las puntuaciones pre-test y pos-test empleando la prueba $t$ de Student para muestras relacionadas, encontrándose que en el grupo experimental aparecían diferencias significativas en la variable autoconciencia $(t=2.889 ; p$ $=.045$ ), diferencias claramente significativas en la variable empatía tanto en la aplicación de EDI ( $t=$ 4.186; $p=.001$ ) como en la sub-escala de ECSE $(t=6,744 ; p=.001)$ y encontrando también diferencias significativas en la variable creatividad $(t=6.114$; $p=.001$ ) (Tabla 4).

Tabla 3

Medias (M) y desviaciones típicas (DT) pre-test y pos-test correspondientes a los grupos de control y experimental

\begin{tabular}{|c|c|c|c|c|c|c|c|c|c|}
\hline \multirow[b]{4}{*}{ Variable } & \multirow{4}{*}{$\begin{array}{l}\text { Instrume } \\
\text { nto }\end{array}$} & \multicolumn{4}{|c|}{ Pretest } & \multicolumn{4}{|c|}{ Postest } \\
\hline & & \multicolumn{2}{|c|}{ Control } & \multicolumn{2}{|c|}{$\begin{array}{l}\text { Experiment } \\
\text { al }\end{array}$} & \multicolumn{2}{|c|}{ Control } & \multicolumn{2}{|c|}{$\begin{array}{l}\text { Experiment } \\
\text { al }\end{array}$} \\
\hline & & & & & & & $\mathrm{D}$ & & \\
\hline & & M & DT & M & DT & M & $\mathrm{T}$ & M & DT \\
\hline & & & 0,48 & 3,2 & 0,31 & 3,3 & 0 & 3,4 & \\
\hline & ECSE & 3,4 & 9 & 94 & 6 & 1 & 42 & 59 & 0,35 \\
\hline Autoconcien & & 3,29 & 0,72 & 3,3 & 0,68 & 3,3 & 0 & 3,5 & 0,56 \\
\hline cia & EDI & 4 & 7 & 25 & 1 & 0 & 70 & 53 & 5 \\
\hline & & & 0,26 & 3,8 & 0,23 & 3,7 & 0 & & 0,83 \\
\hline Empatía & ECSE & 3,69 & 3 & 04 & 7 & 1 & 26 & 4 & 1 \\
\hline & & 12,6 & 3,48 & & 2,98 & 12 & 2 & 15, & 3,88 \\
\hline Creatividad & CREA & 25 & 09 & 9,8 & 42 & 06 & 72 & 8 & 79 \\
\hline
\end{tabular}

Por el contrario, al aplicar la prueba de muestras relacionadas entre la puntuación pre-test y la puntuación pos-test en el grupo control para las tres variables analizadas no se encontraron diferencias significativas en autoconciencia $(t=1,500 ; p=.208)$, ni en empatía (EDI $t=0.105 ; p=.917)$, (ECSE $t=0,542 ; p=.611)$ ni en creatividad $(t=1.542 ; p=.144)$ (Tabla 4$)$. 
Tabla 4

Prueba t de Student para muestras relacionadas de las diferencias pretest-postest en el grupo de control y experimental

\begin{tabular}{ccccccc}
\hline & & \multicolumn{4}{c}{ Control } & \multicolumn{3}{c}{ Experimental } \\
\cline { 3 - 6 } Variable & Instrumento & $\mathrm{t}$ & $\mathrm{p}$ & $\mathrm{t}$ & $\mathrm{p}$ \\
\hline & & & & & \\
Autoconciencia & ECSE & 1,500 & .208 & 2,889 & .045 \\
Empatía & ECSE & .105 & .917 & 4,186 & .0001 \\
Creatividad & CREA & 1,542 & .611 & 6,744 & .001 \\
& & & & & & \\
\hline
\end{tabular}

\section{Discusión y conclusiones}

En base a los resultados podemos comprobar que en el grupo experimental todas las diferencias fueron significativas, tanto para la variable creatividad que experimentó mayor incremento en los valores de puntuación como para la variable empatía, percibiendo una mejora en la capacidad del alunado para adoptar el rol de otro a través de la comprensión del mundo vivencial de sus compañeros. De igual forma se detectan mejoras de atención plena relacionadas con el reconocimiento y modulación de las emociones propias, constatando que la variable autoconciencia experimenta un incremento en las puntuaciones obtenidas tras la aplicación del programa, aunque en menor medida que las variables creatividad y empatía. Al igual que otros estudios realizados en el ámbito educativo donde se demuestran beneficios relacionados con habilidades sociales (De la Fuente Arias, Franco Justo, \& Salvador Granados, 2010) y el rendimiento académico (León, 2009), podemos afirmar la efectividad del programa mindfulness como herramienta útil para el desarrollo de la autoconciencia, la empatía y la creatividad de los estudiantes de Arte Dramático de Galicia. Sería importante replicar el estudio en contextos similares para corroborar estos resultados.

\section{Referencias}

De la Fuente Arias, M., Franco Justo, C. \& Salvador Granados, M. S. (2010). Efectos de un programa de meditación (mindfulness) en la medida de la alexitimia y las habilidades sociales. Psicothema, 22(3),369-375.

http://www.redalyc.org/articulo.oa?id=72714400003

Asuero, M. (2009). Con rumbo propio. Barcelona: Plataforma Editorial.

Baas, M., Nevicka, B., \& Ten Velden, F. S. (2014). Specific mindfulness skills differentially predict creative performance. Personality and Social Psychology Bulletin, 40(9), 1092-1106. http://dx.doi.org/10.1177/0146167214535813

Baer, R. A., Smith, G. T., Hopkins, J., Krietemeyer, J., \& Toney, L. (2006). Using self-report assessment methods to explore facets of mindfulness.

Assessment, 13(1), 27-45.

http://dx.doi.org/10.1177/1073191105283504

Brown, K. W., \& Ryan, R. M. (2003). The benefits of being present: Mindfulness and its role in psychological well-being. Journal of Personality and Social Psychology, 84(4),

822. http://dx.doi.org/10.1037/00223514.84.4.822

Carboni, J. A. (2012). The impact of mindfulness training on hyperactive behaviors demonstrated by elementary age children with a diagnosis of attention deficit hyperactivity disorder.

http://scholarworks.gsu.edu/cps_diss/72

Carnicke, S. M. (2009). Stanislavski in focus an acting master for the twenty-first century. Londres: Galaxia.

Chambers, R., Gullone, E., \& Allen, N. B. (2009). Mindful emotion regulation: An integrative review. Clinical Psychology Review, 29(6), 560-572. http://dx.doi.org/10.1016/j.cpr.2009.06.005

Chéjov, M. (1999). Sobre la técnica de la actuación. Barcelona: Alba Editorial.

Corbalan, J., Martínez, F., \& Donolo, D. (2003). Manual test CREA inteligencia creativa una medida cognitiva de la creatividad. Madrid: Tea Ediciones.

C. K., Nijstad, B. A., Baas, M., Wolsink, I., \& Roskes, M. (2012). Working memory benefits creative insight, musical improvisation, and original ideation through maintained task-focused attention. Personality \& Social Psychology Bulletin, 38(5), 656-669. http://dx.doi.org/10.1177/0146167211435795 http://psp.sagepub.com/content/38/5/656.short

Davis, M. H. (1994). Empathy: A social Pschological approach. New York: Westview Press.

Duval, S., \& Wicklund, R. A. (1972). A theory of objective self awareness. Oxford, England: Academic Press.

Gardner, H., \& Nogués, M. T. M. (1998). Inteligencias múltiples. Barcelona: Paidós.

Kabat-Zinn, J. (2005). Coming to our senses: Healing ourselves and the world through mindfulness Hachette. United States: Hyperion Books.

Layton, W., \& Esteve, J. A. L. (1995). ¿ Por qué? trampolín del actor. Madrid: Editorial Fundamentos.

León, B. (2009). Atención plena y rendimiento académico en estudiantes de enseñanza secundaria. European Journal of Education and Psychology, 1(3), 17-26.

http://www.ejep.es/index.php/journal/article/viewArti cle/13\#

Meyerhold, V. E. (1975). Teoría teatral. Madrid: Editorial Fundamentos.

Motos Teruel, T., \& Tejedo, F. (1987). Prácticas de dramatización. Barcelona: Editorial Ñaque.

Repetto, E. (2009). Formación en competencias socioemocionales (libro del formador CD). Madrid: Editorial La Muralla.

Rovira, D. P., \& Perosanz, J. J. I. (1998). Validez y fiabilidad de una escala de empatía e identificación con los personajes. Psicothema, 10(2), 423-436. http://dialnet.unirioja.es/servlet/articulo?codigo=2013 636

Scheier, M. F., \& Carver, C. S. (1985). Optimism, coping, and health: Assessment and implications of generalized outcome expectancies. Health Psychology,4(3),219.

http://psycnet.apa.org/doi/10.1037/h0076760 
Serrano, R. (2004). Nuevas tesis sobre Stanislavski: Fundamentos para una teoría pedagógica. Buenos Aires: Atuel.

Sibinga, E. M., Perry-Parrish, C., Chung, S., Johnson, S. B., Smith, M., \& Ellen, J. M. (2013). School-based mindfulness instruction for urban male youth: A small randomized controlled trial. Preventive Medicine, 57(6), 799-801.

http://dx.doi.org/10.1016/j.ypmed.2013.08.027

Siegel, D. J. (2015). The developing mind: How relationships and the brain interact to shape who we are. New York: Guilford Publications.

Stanislavski, K. (2013). Mi vida en el arte. Barcelona: Alba Editorial. 